\title{
An update on osteoporosis
}

\author{
Oana Bodean*, Octavian Munteanu* **, Diana Voicu*, Luciana Arsene*, \\ Ana Uzunov*, Florina Pauleț*, Monica Cîrstoiu* *** \\ *Obstetrics and Gynecology Department, Emergency University Hospital Bucharest \\ **Anatomy Department, "Carol Davila" University of Medicine and Pharmacy, Bucharest \\ ***Obstetrics and Gynecology Department, "Carol Davila" University of Medicine \\ and Pharmacy, Bucharest
}

Correspondence to: Octavian Munteanu, MD, PhD, Obstetrics and Gynecology Department, Emergency University Hospital Bucharest, 169 Splaiul Independentei Street, Code 050098, Bucharest, Romania, Mobile phone: +40722650 092, E-mail: octav_munteanu@yahoo.com

\begin{abstract}
With the growth of the aging population worldwide, osteoporosis represents a serious health problem, which implies higher morbidity and costs, despite the continuous advances in treatment options. It involves both sexes, although menopausal women are mainly affected. This article is a brief review of literature on current osteoporosis treatment options.

Keywords: osteoporosis, bone loss, bone fractures, osteoporosis treatment
\end{abstract}

\section{Introduction}

By definition, osteoporosis is characterized by low bone density and deterioration of bone architecture, with an increased risk of fragility fractures. According to the degree of bone density loss, three situations come to discussion: osteopenia, osteoporosis, and severe osteoporosis. World Health Organization defines osteopenia when bone density drops below one standard deviation (-1.0 to -2.5 SD) compared to normal young adult bone density ( $>-1.0$ ); osteoporosis occurs when bone density drops < -2.5 SD; severe osteoporosis is defined when a fragility fracture occurs on osteoporotic bone [1].

Approximately 200 million women worldwide are affected by different degrees of osteoporosis. Interestingly, Riggs et al. reported that the total annual incidence of osteoporotic bone fractures in women of all ages exceeds the annual incidence of acute heart failure in women over 29 years old, of stroke in women over 30 and of breast cancer in women of all ages [2]. Rosen et al. observed that after the age of 50, the incidence of vertebral fractures increases dramatically, followed by the incidence of hip fractures and by the constant increase of forearm fractures [3].

In their study on 2725 postmenopausal women, Lindsay et al. calculated the relative risk of subsequent vertebral fractures depending on preexisting vertebral fractures and found that the relative risk is as high as 7.3 in women with $\geq 2$ previous fractures even in the first year. The consequences of osteoporosis on the patients' quality of life are even worse 
when it comes to hip fractures [4]. Cooper et al. reported that at one year after a hip fracture, $80 \%$ of the patients still had the impairment to perform some routine activities by themselves, $40 \%$ could not walk by themselves, $30 \%$ had permanent sequelae and $20 \%$ of the patients died in the first year after osteoporotic hip fracture [5].

In a larger study on 6459 postmenopausal women of 55 to 81 years old, with a follow-up period of 3.8 years, Cauley et al. calculated the relative risk of death correlated to clinical bone fractures. The highest relative risk was reported for spine fractures $(R R=8.6)$ and for hip fractures (RR=6.7) [6]. Because osteoporosis has such an impact on the quality of life, early diagnosis and proper treatment are mandatory.

\section{Diagnosis of osteoporosis}

Early recognition of osteoporosis is of main importance, because the disease occursina wide variety of patients with comorbidities. Primary osteoporosis occurs with aging and is of type I (in patients $>$ 50-70 years old) and type II (in patients $>70$ years old). Secondary osteoporosis comprises a large number of disturbances: endocrine pathology, malignancies [7-10], side effects of certain medical treatments, longterm immobilization, chronic kidney disease [11], and chronic inflammatory pathology. Risk factors are genetic, gender-related, ethnicity-related, dietary-related, smoking, sedentariness, certain chronic therapy (corticosteroids, heparin, antacids, and thyroid medication) [12].

\section{Clinical diagnosis}

Most patients are asymptomatic. Menopausal women encounter pain or notice their pain only when dealing with complications of osteoporosis. There are multiple sources of pain in osteoporosis: local congestion and hypoxia, micro-trabecular fractures, compression fractures, stress fractures, rib pain, paravertebral and inter-scapular-vertebral muscle contractions, arthrosis due to regional bone deformities. Acute pain is usually caused by micro-fractures due to minor trauma, sudden movements, or mild to moderate efforts. It usually has a sudden onset and a high intensity and is alleviated by non-steroidal anti-inflammatory (NSAIDs) medication and rest. Chronic pain installs insidiously, has a slow progressive evolution, becomes permanent and it limits the patient's effort capacity. It is usually located to the spine and is augmented by the orthostatic position. It associates fatigability, it can disrupt the patient's sleep, it can be initially alleviated with NSAIDs, but these become ineffective in time [13].

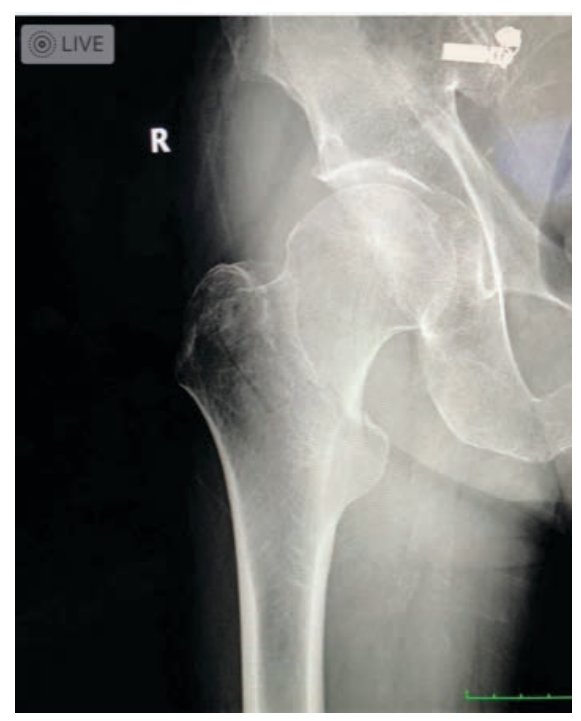

Hip joint

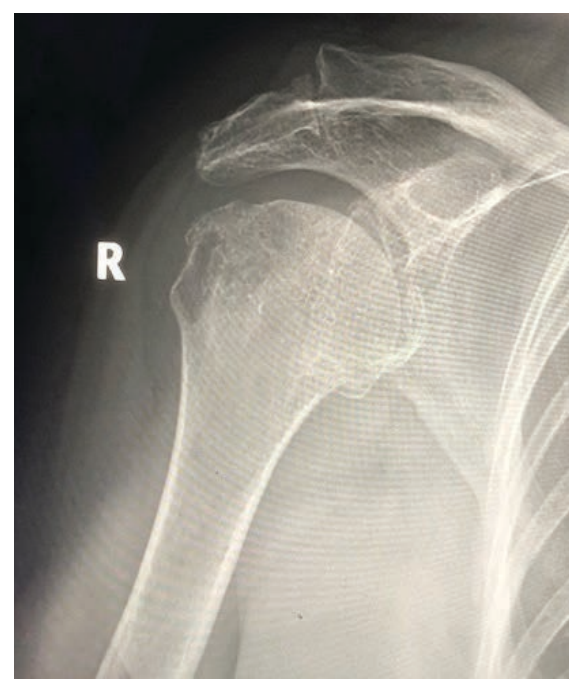

Shoulder joint

Fig. 1 X-rays in patients diagnosed with osteoporosis - note important bone loss 


\section{Paraclinical diagnosis}

Bone mineral density (BMD) measurement is the main tool to assess bone strength (Z-scores), but in clinical practice, there are also other skeletal and non-skeletal factors, which contribute to the overall fracture risk. These factors are included in the Fracture Risk Assessment Tool (FRAX), which is a computer algorithm made to estimate the 10-year fracture risk probability for a major osteoporotic fracture (hip, spine, humerus, and wrist).

Other imaging techniques that can be used are: dual X-ray absorptiometry (DXA), quantitative computed tomography (QTC), quantitative ultrasound (QUS) [14].

Biochemical markers of bone formation and bone resorption can also be used. Alkaline phosphatase, osteocalcin, P1NP (Procolagen${ }_{1} \mathrm{~N}$ Peptide) are markers of bone formation. Acid phosphatase, pyridinium crosslinks, deoxypyridinoline (DPD), and collagen type 1 cross-linked C-telopeptide (CTX) are some of the novel used biochemical markers of bone resorption. The only enzyme with a clinical importance in bone pathology is alkaline phosphatase. The other biochemical markers are used in treatment monitoring, in identifying patients at risk of fragility fractures and in verifying patients' adherence to treatment. The clinical application and use of these biomarkers have certain limitations: they cannot differentiate between cortical and trabecular bone, they can reflect different stages of bone formation and resorption, they cannot reflect osteocyte activity, they give different response according to various therapeutic agents, they have a wide variability of methods used for the same marker and they also have a wide biological variability [15].

\section{Treatment of osteoporosis}

The aim of the treatment of osteoporosis is to reduce moderate and high fracture risk. When using FRAX, the estimate fracture risk is assessed according to the patient's risk factors, epidemiological data, and medication taken. The online system is linked to guidelines of the National Osteoporosis Guideline Group, which displays a treatment threshold and aids management decisions. Treatment is recommended to patients with a hip fracture risk > 3\% and with a > 20\% risk for another osteoporotic fracture [16].

Screening for osteoporosis is recommended in patients over 65 years old and/ or in women with a fracture risk higher than that of a 65 -yearold Caucasian woman with no risk factors [13].

\section{Non-pharmacological management and prevention}

An individual's bone mineral density is determined by many factors, such as heredity and modifiable factors (diet, smoking, exercise, endocrine status). Intrauterine environmental factors (maternal nutrition, vitamin $D$ levels) influence the epigenetic mechanisms underlying the development of the fetal skeleton. Peak bone density is achieved at the age of 30 and is a key predictor of osteoporotic fractures at older age. Several studies on children concluded that maternal $25(\mathrm{OH}) \mathrm{D}$ during late pregnancy was associated with decreased bone mineral content in their children at the age of 9 and also that regular physical exercise in childhood improves bone mass. A healthy lifestyle is recommended further on, as bone density and muscle mass begin to decline after the age of 30. An ideal body mass index should be encouraged, keeping in mind that both obesity and a low BMI represent risk factors for osteoporosis and fragility bone fractures. The elderly patients have the highest risk of fractures, therefore prevention of falling and mobility improvement strategies are very important [17].

\section{Pharmacological treatment}

Before introducing medical treatment, the patient must be given an appropriate diet, with an optimal calcium and vitamin $D$ intake. 


\section{Calcium and vitamin D}

Recent studies suggest that there is a possible association between calcium supplements and heart attack risk. Therefore, the National Osteoporosis Foundation recommends that calcium must be taken primarily from food sources, with a total daily intake of 1000mg-1200mg (total amount from both food and supplements). Vitamin D daily intake should be 400-80oui [18].

Selective Estrogen Receptor Modulators (SERMs)

The most important factor contributing to bone loss in post-menopause is the decrease in estrogen. Hormonal replacement therapy (HRT) is risky and controversial and is only used with caution. It increased the risk for breast cancer, cardiovascular and venous thromboembolic events (VTE). Unopposed estrogen replacement increased the risk for stroke. Before using HRT, the risks and benefits must be clear and must be kept in mind that withdrawal from estrogen produces bone loss [17]. A safer option are SERMs, which have an agonist effect on the estrogen receptors in bone and an antagonist effect on the estrogen receptors in breast and brain. Studies proved that raloxifene 6omg daily reduces the risk of vertebral fractures by $30 \%$ over 3 years (The MORE Study). Unfortunately, it increases the risk for VTE and is contraindicated for pre-menopausal women. NICE recommends the use of raloxifene in secondary prevention of osteoporotic bone fractures in postmenopausal women who are intolerant or have contraindications for bisphosphonates. It can also be used in women with a high risk for breast cancer and it has no effects on the endometrium [19].

\section{Bisphosphonates}

Bisphosphonates are the treatment of choice in osteoporosis for menopausal women. They are synthetic analogues of naturally occurring inorganic pyrophosphate, have a strong affinity for mineral, and act at sites of active bone resorption and new bone formation.
They are eventually covered by the new bone and their action lasts for years. The most common side effects of oral bisphosphonates are gastrointestinal symptoms. Other side effects are osteonecrosis of the jaw and atypical femoral fractures [17]. Balmetal., Woo etal., and other authors report atypical fractures of the femoral diaphysis in postmenopausal women taking alendronate $[20,21]$. Bisphosphonates reduce the risk of typical femur fractures after 5 years treatment but increases the risk of atypical fractures after 5 years. Therefore, the length of the treatment is a matter of debate. The major benefits of bisphosphonates therapy are low risk for breast, colorectal, gastric cancer risk, low stroke risk and a decrease in overall mortality. The treatment is recommended to menopausal women with a T-score $<2.5$ at femoral cervix after 3-5 years of prophylaxis for vertebral fractures and to women with a vertebral fracture and a T-score of -2.0 [22].

\section{Denosumab}

Denosumab is a human anti-RANKL monoclonal antibody, which helps inhibiting osteoclastogenesis. Recommended dosage is 6omg subcutaneous injection at every 6 months. It significantly reduces vertebral, hip, and non-vertebral fracture risk in postmenopausal women by $68 \%, 40 \%$, and $20 \%$ after 3 years of administration. It is contraindicated in hypocalcemia; therefore, patients should have proper calcium and vitamin $D$ levels prior to and during treatment. NICE recommends denosumab for primary prevention of osteoporotic fractures in postmenopausal women if bisphosphonates are contraindicated [23].

The use of strontium ranelate was restricted only to very few selected cases with severe osteoporosis, with contraindications for all other options, because of its high risk of severe cerebrovascular disease and thromboembolic events [17].

\section{New developments}

A monoclonal anti-sclerostin antibody and a selective cathepsin $\mathrm{K}$ inhibitor have been 
tested in clinical trials with encouraging results, but conclusions are currently under way [17].

\section{Conclusions}

Osteoporosis remains an important public health issue, with its prevalence increasing with the ageing population, despite the important advances in diagnosis and treatment. FRAX remains an important tool and treatment should be given to all patients who score for an increased fracture risk, according to specific characteristics. Usually, bisphosphonates followed by denosumab are the drugs of choice for postmenopausal women with moderate to high fracture risk. SERMs are an option for younger postmenopausal women at a greater risk for vertebral than hip fractures. Prevention strategies should be applied as early as possible, with a healthy lifestyle and a balanced nutrition.

\section{Acknowledgement}

The authors have participated equally to this article.

\section{Conflict of interest}

The authors have no conflicts of interest to declare.

\section{References}

1. Kanis JA, Melton LJ, Christiansen C, Johnston CC, Khaltaev N. The diagnosis of osteoporosis. Journal of bone and mineral research. 1994; 9(8):1137-1141.

2. Riggs BL, Melton Iii LJ. The worldwide problem of osteoporosis: insights afforded by epidemiology. Bone. 1995; 17(5):S505-S511.

3. Rosen CJ, Compston JE, Lian JB. ASBMR primer on the metabolic bone diseases and disorders of mineral metabolism. 2009, John Wiley \& Sons.

4. Lindsay R, Silverman SL, Cooper C, Hanley DA, Barton I, Broy SB, Stracke H. Risk of new vertebral fracture in the year following a fracture. Jama. 2001; 285(3):320-323.

5. Cooper $\mathrm{C}$. The crippling consequences of fractures and their impact on quality of life. The American Journal of Medicine. 1997; 103(2):S12-S19.

6. Cauley JA, Thompson DE, Ensrud KC, Scott JC, Black D. Risk of mortality following clinical fractures. Osteoporosis International. 2000; 11(7):556-561.
7. Sinescu RD, Niculae A, Peride I, Vasilescu F, Bratu OG, Mischianu DL, Checheriță IA. Uterus neuroendocrine tumor-a severe prognostic factor in a female patient with alcoholic cirrhosis undergoing chronic hemodialysis. Rom J Morphol Embryol. 2015; 56(2):601-605.

8. Bratu O, Mischianu D, Spanu D, Barla R, Hoara P, Constantinoiu S. Paraneoplastic Syndrome in Primitive Retroperitoneal Tumours. Chirurgia. 2013; 108:26-31.

9. Niculae A, Peride I, Vinereanu V, Radulescu D, Bratu OG, Geavlete BF, Checherita IA. Nephrotic syndrome secondary to amyloidosis in a patient with monoclonal gammopathy with renal significance (MGRS). Rom J Morphol Embryol. 2017; 58(3):1065-1068.

10. Niculae A, Peride I, Marinescu-Paninopol A, Vrabie CD, Ginghina O, Jecan CR, Bratu OG. Renal artery bilateral arteriosclerosis cause of resistant hypertension in hemodialysed patients. Rom J Morphol Embryol. 2016; 57(2):591-594.

11. Checherita IA, Smarandache D, Radulesc D, Peride I, Bratu O, Ciocâlteu A, Lascar I. Calcific uremic arteriolopathy in hemodialyzed patients. Chirurgia. 2013; 108(5):736-740.

12. Radulescu D, Stroescu A, Pricop C, Geavlete B, Negrei C, Bratu O, Vacaroiu IA. Vitamin K Influence on Cardiovascular Mortality in Chronic Hemodialysed Patients. Revista de Chimie. 2017; 68(1):52-54.

13. Kling JM, Clarke BL, Sandhu NP. Osteoporosis prevention, screening, and treatment: a review. Journal of Women's Health. 2014; 23(7):563-572.

14. Warriner AH, Saag KG. Osteoporosis diagnosis and medical treatment. Orthopedic Clinics. 2013; 44(2):125135.

15. Nishizawa Y, Nakamura T, Ohta H, Kushida K, Gorai I, Shiraki M, Ichimura S. Guidelines for the use of biochemical markers of bone turnover in osteoporosis (2004). Journal of Bone and Mineral Metabolism. 2005; 23(2):97-104.

16. Kanis JA, McCloskey EV, Johansson H, Oden A, Ström 0 , Borgström F. Development and use of FRAX® in osteoporosis. Osteoporosis International. 2010; 21(2):407-413.

17. Daroszewska A. Prevention and treatment of osteoporosis in women: an update. Obstetrics, Gynaecology \& Reproductive Medicine. 2012; 22(6):162-169.

18. Li K, Kaaks R, Linseisen J, Rohrmann S. Associations of dietary calcium intake and calcium supplementation with myocardial infarction and stroke risk and overall cardiovascular mortality in the Heidelberg cohort of the European Prospective Investigation into Cancer and Nutrition study (EPIC-Heidelberg). Heart. 2012; 98(12):920-925.

19. Stevenson M, Jones ML, De Nigris E, Brewer N, Davis S, Oakley J. A systematic review and economic evaluation of alendronate, etidronate, risedronate, raloxifene and teriparatide for the prevention and treatment of postmenopausal osteoporosis. 2005.

20. Baim S, Miller PD. Perspective: assessing the clinical 
utility of serum CTX in postmenopausal osteoporosis and its use in predicting risk of osteonecrosis of the jaw. Journal of Bone and Mineral Research. 2009; 24(4):561574.

21. Woo SB, Hellstein JW, Kalmar JR. Systematic review: bisphosphonates and osteonecrosis of the jaws. Annals of Internal Medicine. 2006; 144(10):753-761.

22. Park-Wyllie LY, Mamdani MM, Juurlink DN, Hawker GA, Gunraj N, Austin PC, Laupacis A. Bisphosphonate use and the risk of subtrochanteric or femoral shaft fractures in older women. Jama. 2011; 305(8):783-789.

23. Grimer RJ. Denosumab: a drug that you may be hearing a lot more about. Bone\&Joint 360. 2013; 2(5):43-44. 